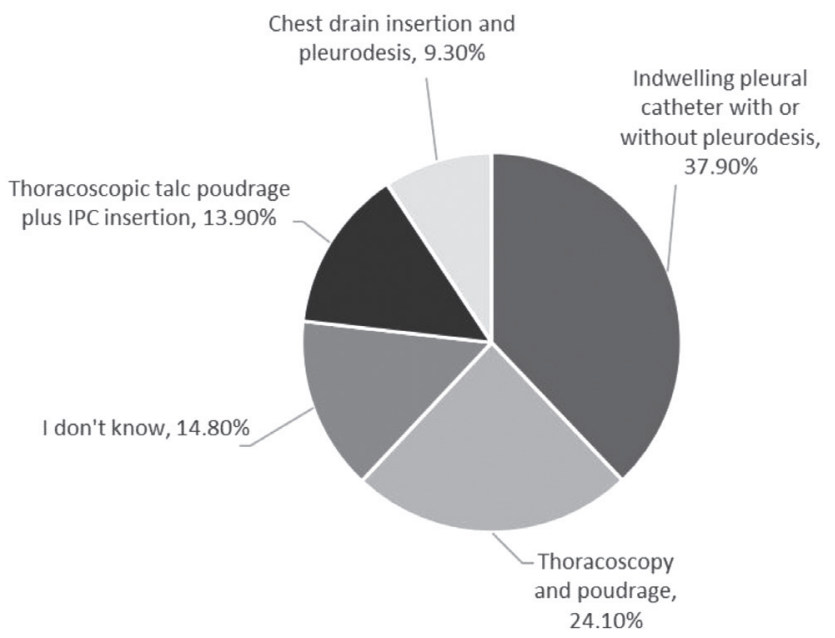

Abstract P7 Figure 1 Which intervention most improves quality of life in malignant pleural effusion? $(n=108)$

Conclusion Shortness of breath and chest pain ranked highly in the perspective of HRQOL with shortness of breath a key factor in offering intervention. There is a lack of consensus on the ideal treatment to maximise HRQOL, which may reflect the paucity of data. Robust clinical trial evidence on HRQOL outcomes is therefore required to guide management decisions of patients with MPE. This should be complemented by a patient survey to ascertain differences in clinician and patient perspectives of quality of life and care.

\section{P8 NEGATIVE PLEURAL BIOPSIES - DO WE NEED EARLY FOLLOW UP AND IMAGING?}

${ }^{1} S$ Leyakathali Khan, ${ }^{2} \mathrm{~B}$ Ganaie, ${ }^{1} \mathrm{M}$ Haris, ${ }^{2} \mathrm{M}$ Munavvar. 'Royal Stoke University Hospital, Stoke on Trent, UK; ${ }^{2}$ Lancashire Teaching Hospital NHS trust, Preston, UK

\subsection{6/thoraxjnl-2016-209333.151}

Background Primary pleural or secondary malignancy is a common cause of pleural effusion. The incidence is about 200000 cases per year. Pleural biopsy remains gold standard investigation of choice. Those with negative biopsies are either discharged or have follow up depending on the multidisciplinary team decision. Objective To review the outcome of all patients with negative pleural biopsy including any follow up imaging for up to two years.

Methods Retrospective analysis of 162 consecutive patients who underwent video-assisted thoracoscopic surgery (VATS) (100) and local anaesthetic thoracoscopy (62) between January 2011 and December 2012 across two large UK tertiary referral centres. Patients referred from peripheral centres were excluded.

Results Of the 162 patients, male:female ratio was 109/53; average age was 69 . Pleural biopsy histology was malignant in $63 \%$ (100); mesothelioma 43\%, lung cancer 35\%, extra pulmonary 22\%. Granulomatous inflammation 6\% (9). Benign 31\% (53); chronic inflammation 28, fibrosis/thickening 8, reactive 13 and others 4. See Table 1.

Of the 53 benign, 11 (21\%) developed malignancy before 2 years. $6(55 \%)$ required repeat biopsy. Patients alive at the end of 2 years -1 Malignant and 26 benign of the 53 .
Abstract P8 Table 1 VATS and Thoracoscopy Pleural Biopsies

\begin{tabular}{llll}
\hline & Centre 1 & Centre 2 & \\
\hline Data from 2011 to 2012 & VATS & Thoracoscopy & Total, $\mathrm{n}(\%)$ \\
\hline Number & 100 & 62 & 162 \\
Malignant on biopsy & 60 & 40 & $100963 \%)$ \\
Granulomatous & 5 & 4 & $9(6 \%)$ \\
Benign & 35 & 18 & $53(31 \%)$ \\
- Chronic inflammation & 16 & 12 & 28 \\
- Fibrosis/thickening & 8 & 0 & 8 \\
- Reactive & 8 & 4 & 12 \\
- Others & 3 & 2 & 5 \\
Malignant progression & 8 & 3 & $11(21 \%)$ \\
\hline
\end{tabular}

Imaging performed during 2 years follow up at 6, 12, and 24 months: plain chest radiograph 22 (42\%), 12 (23\%), 13 (25\%) and CT scan was done at 5 (9\%), 8 (16\%), 2 (4\%) respectively. Conclusion Our data suggests that $21 \%$ of patients were diagnosed as malignant within 2 years of initial negative biopsy, which is higher than expected. ${ }^{1}$ There is a need for early follow up and imaging in patients with negative pleural biopsy. Further studies are required to establish the follow up interval and imaging modality.

\section{REFERENCE}

1 Janssen J, Ramlal S, Mravunac M. The long-term follow up of exudative pleural effusion after nondiagnostic thoracoscopy. J Bronchol 2004;11(3):169-174.

\section{P9 $\quad$ THE UTILITY OF P16 FISH IN DIFFERENTIATING MALIGNANT MESOTHELIOMA AND BENIGN MESOTHELIAL PROLIFERATIONS}

A Chaturvedi, J Holme, R Shah, P Taylor, M Evison. University Hospital of South Manchester, Manchester, UK

\subsection{6/thoraxjnl-2016-209333.152}

Introduction One of the commonest genetic abnormalities in malignant mesothelioma is deletion of the 9 p21 locus which harbours the p16/CDKN2A gene. Homozygous deletion of $\mathrm{p} 16 /$ CDKN2A can be identified with Fluorescence in situ hybridization (FISH) and may be a useful diagnostic tool where there is difficulty separating malignant from benign mesothelial cell proliferations, e.g. where a lack of invasion into adipose tissue prevents a confident diagnosis of mesothelioma.

Methods The University Hospital of South Manchester is a regional mesothelioma centre in the North West of England. p16 FISH has been in clinical use since 2013 for cases of abnormal mesothelial cell proliferation without conclusive evidence of malignancy. This retrospective study analysed the diagnostic performance of p16 FISH using clinical follow-up and post-mortem studies to clarify final diagnoses.

Results 75 pathological samples underwent p16 FISH analysis 2013-2015; 16 cytology samples (14 pleural fluid, 2 ascitic fluid), 36 VATS pleural biopsies, 16 local anaesthetic thoracoscopy pleural biopsies and 7 percutaneous pleural biopsies. There was one failed test. A final diagnosis based on subsequent definitive pathological sampling, definitive radiological surveillance or post-mortem findings were available for $99 \%$ of patient $(74 / 75)$. 71 patients were ultimately proven to have mesothelioma (39 epithelioid, 13 sarcomatoid, 7 biphasic and 12 NOS), 2 patients were diagnosed benign pleural disease and 1 with metastatic lung cancer. The diagnostic performance of p16 FISH was as follows: 